\title{
BMJ Open Anxiety and clinical outcomes of patients with acute coronary syndrome: a meta-analysis
}

Jie Li, ${ }^{1}$ Feng $\mathrm{Ji}^{2},{ }^{\text {Junxian Song }},{ }^{3}$ Xiangyang Gao, ${ }^{4}$ Deguo Jiang, ${ }^{1}$
Guangdong Chen, ${ }^{1}$ Suling Chen,,${ }^{1}$ Xiaodong Lin,,${ }^{5}$ Chuanjun Zhuo (D) ${ }^{1,2,6}$

\section{ABSTRACT}

Objectives Anxiety has been suggested to be associated with poor outcomes in patients with acute coronary syndrome (ACS). However, results of previous follow-up studies were inconsistent. The aim of this meta-analysis was to evaluate the association between anxiety and clinical outcomes in patients with ACS, and to investigate the potential role of depression underlying the above association.

Design A meta-analysis of prospective follow-up studies.

Setting Hospitals.

Participants Patients with ACS.

Interventions We included related prospective followup studies up through 20 July 2019 that were identified by searching PubMed and Embase databases. A random-effect model was used for the meta-analysis. Anxiety was evaluated by validated instruments at baseline.

Primary and secondary outcome measures We determined the association between anxiety and risks of mortality and adverse cardiovascular events (MACEs) in patients with ACS.

Results Our analysis included 17 studies involving 39038 patients wqith ACS. Anxiety was independently associated with increased mortality risk (adjusted risk ratio (RR) $1.21,95 \% \mathrm{Cl} 1.07$ to $1.37, \mathrm{p}=0.002$ ) and MACEs (adjusted RR 1.47, $95 \% \mathrm{Cl} 1.24$ to 1.74 , $\mathrm{p}<0.001$ ) in patients with ACS. Subgroup analyses showed that depression may at least partly confound the association between anxiety and poor outcomes in patients with ACS. Adjustment of depression significantly attenuated the association between anxiety and MACEs (adjusted RR 1.25, 95\% Cl 1.04 to 1.52, $p=0.02$ ). Moreover, anxiety was not significantly associated with mortality risk after adjusting for depression (adjusted RR $0.88,95 \% \mathrm{Cl} 0.66$ to $1.17, \mathrm{p}=0.37$ ).

Conclusions Anxiety is associated with increased risk of mortality and MACEs in patients with ACS. However, at least part of the association may be confounded by concurrent depressive symptoms in these patients.

\section{INTRODUCTION}

Despite significant improvement in the management of patients with coronary artery disease (CAD) in recent decades, CAD, particularly acute coronary syndrome

\section{Strengths and limitations of this study}

- A comprehensive meta-analysis including 17 studies.

- Only prospective follow-up studies were included.

- Only studies with adjustment of confounding factors were included.

- Studies published in languages other than English were omitted.

- Different instruments were used for anxiety diagnosis in the included studies.

(ACS), remains one of the leading causes of death worldwide. ${ }^{1-3}$ ACS, including acute myocardial infarction (MI) and unstable angina (UA), refers to a category of severe CAD characterised by acute rupture of unstable atherosclerotic plaques and subsequent obstruction of the coronary artery lumen. ${ }^{4}$ Patients with ACS not only suffer from severe clinical symptoms of chest pain, but are also vulnerable to fatal complications such as malignant arrhythmia and haemodynamic instability. ${ }^{4}$ Moreover, invasive procedures, primarily including percutaneous coronary intervention or coronary artery bypass graft, are frequently performed in these patients. ${ }^{5}$ All of the above factors may contribute to the pathogenesis of affective disorders in these patients, such as depression and anxiety. ${ }^{6}$

Previous studies have confirmed that depression independently predicts poor clinical outcomes in patients with CAD. ${ }^{7}$ However, the influence of anxiety on prognosis of CAD patients, particularly for those with ACS, is poorly understood. ${ }^{8}$ Although the potential prognostic efficacy of anxiety for patients with ACS has been previously evaluated, results of these studies are inconsistent. $^{9-25}$ Some studies indicated that anxiety is a risk factor of poor prognosis in patients with ACS, ${ }^{911} 12202325$ while others did not support this finding. ${ }^{10} 13-19212224$ 
Although two previous meta-analyses found that patients with ACS with anxiety may have a higher risk of mortality and other adverse outcomes compared with those without anxiety, ${ }^{26}{ }^{27}$ these conclusions were mainly based on studies with univariate analyses, and one of the meta-analyses included a high risk of publication bias. ${ }^{26}$ However, many prospective studies with multivariable analyses have been performed to evaluate the effect of anxiety on prognosis in patients with ACS, ${ }^{16-25}$ providing rationale to perform an updated meta-analysis. Considering that anxiety and depression are highly correlated psychological disorders, ${ }^{28}$ it is important to determine the extent to which the association between anxiety and prognosis of patients with ACS is independent of depression. Accordingly, we performed an updated meta-analysis to evaluate the potential prognostic influence of anxiety on adverse clinical outcomes in patients with ACS. Moreover, we aimed to explore whether concurrent depression confounds the association between anxiety and adverse outcomes in patients with ACS.

\section{METHODS}

This study was designed as a meta-analysis of prospective observational studies, and was performed in accordance with the Meta-analysis of Observational Studies in Epidemiology ${ }^{29}$ and Cochrane's Handbook ${ }^{30}$ guidelines.

\section{Literature search}

PubMed and Embase were searched for relevant records with the combination of the following terms: (1) "anxiety" OR "tension" OR "post-traumatic stress disorder" OR "panic" OR "phobia” OR "phobic" OR "worry"; (2) "myocardial infarction" OR "acute coronary syndrome" OR ACS OR "unstable angina"; and (3) "cohort" OR "cohorts" OR "follow-up" OR "followed" OR "retrospective" OR "prospective" OR "retrospectively" OR "prospectively" OR "mortality" OR "prognosis" OR "survival" OR "adverse events". Only human studies published in English were included. The reference lists of original and review articles were manually screened as a supplementation. The final search was performed on 20 July 2019. The full-search strategy for PubMed was presented in online supplementary file 1.

\section{Study selection}

Studies were included according to the following criteria: (1) full-length article in English; (2) designed as prospective follow-up studies with a minimal follow-up duration of 1 year; (3) included at least 100 patients with ACS; (4) anxiety assessed within 3 months of the onset of ACS as exposure of interest; (5) documented the incidence of mortality (all cause or cardiovascular) and/or major adverse cardiovascular events (MACEs) in patients with and without anxiety at baseline and (6) reported the multivariable adjusted risk ratios (RRs) and their corresponding 95\% CIs for mortality and/ or MACEs outcomes in patients with anxiety compared with those without anxiety. MACEs were defined as a composite outcome of cardiac death, non-fatal MI, cardiac rehospitalisation, recurrence of ACS and repeated coronary revascularisation. The diagnosis of anxiety was consistent with the criteria of the original articles. For repeated reports of the same cohort, the latest studies with the longest follow-up duration were included.

\section{Data extraction and quality evaluation}

Two authors independently performed the literature search, data extraction and quality assessment. If discrepancies occurred, they were resolved by consultation with the corresponding author. A predefined form was used for data extraction. The extracted data included: (1) first author, location and design of the study; (2) number, mean age, gender and diagnosis of the patients; (3) diagnostic tools for anxiety and number of patients with anxiety; (4) follow-up durations, outcomes reported, number of patients with outcomes and variables adjusted and (5) outcome data for the mortality/ MACEs risk in patients with ACS with anxiety compared with those without anxiety as presented in RRs and 95\% CIs. Study quality was evaluated using the NewcastleOttawa Scale. ${ }^{31}$ This scale ranges from 1 to 9 stars and assesses quality of the individual study according to the following three aspects: selection of the study groups; comparability of the groups and ascertainment of the outcome of interest.

\section{Statistical analyses}

Data of RRs and their corresponding SEs were estimated from $95 \%$ CIs or $\mathrm{p}$ values, and then logarithmically transformed to stabilise the variance and to normalise the distribution of the data. ${ }^{30}$ For the two studies where the OR was described, ${ }^{92}$ we converted data to RR for meta-analysis $\quad(\mathrm{RR}=\mathrm{OR} /([1-\mathrm{pRef}]+[\mathrm{pRef} \times \mathrm{OR}]) \quad$ as previously described, ${ }^{32}$ where pRef is the prevalence of the outcome in the reference group. The Cochrane's $Q$ test was performed to evaluate the heterogeneity among studies, as well as the calculation of the $\mathrm{I}^{2}$ statistic. ${ }^{33} \mathrm{An}$ $\mathrm{I}^{2}>50 \%$ indicated significant heterogeneity. A randomeffect model was used for the meta-analysis since this model could incorporate the potential heterogeneity of the included studies and provide a more generalised result. ${ }^{30}$ Sensitivity analyses, which remove studies one at a time, were performed to evaluate the stability of the results. ${ }^{34}$ Subgroup analysis was performed to evaluate whether subtypes of ACS or adjusting for depression influenced the results. Risk of publication bias was assessed by funnel plots, complemented with the Egger regression asymmetry test. ${ }^{35}$ RevMan (V.5.1; Cochrane Collaboration, Oxford, UK) and STATA software (V.12.0; StataCorp) were used for the statistical analyses. 
Studies identified through database search $(n=1392)$

Studies excluded based on title and abstract $(n=1333$ )

Not relevant studies

Not prospective follow-up studies

Review articles, letters, or preclinical studies

Potentially relevant studies $(n=59)$

Studies excluded based on full-text review $(n=42)$

Not prospective follow-up studies $(n=6)$

Not in patients with ACS ( $n=12$ )

Not include anxiety at baseline as exposure $(n=4)$

Outcomes of interest not reported $(n=8)$

Adjusted data not available $(n=7)$

Studies with follow-up < 1 year $(n=2)$

Repeated report of the same cohort $(n=3)$

Studies included in meta-analysis $(n=17)$

Anxiety and adverse outcomes in acute coronary syndrome

Figure 1 Flow chart of database search and study

identification. ACS, acute coronary syndrome.

\section{Patient and public involvement}

No patients were involved in developing the research question or the outcome measures, and no patients were involved in planning the design or implementation of the study. Furthermore, no patients were asked to advise on the interpretation or write-up of the results. Since this study used aggregated data from previous publications, it is not easy to directly disseminate the results of the research to the study participants.

\section{RESULTS}

\section{Results of study selection}

The database search and study identification are shown in figure 1. A total of 1392 studies were obtained via literature search, of which 17 studies were finally included. ${ }^{9-25}$

\section{Study characteristics and quality evaluation}

The characteristics of the studies are shown in table 1 . Overall, this meta-analysis included 39038 patients with ACS. ${ }^{9-25}$ All studies were performed in countries from North America or Europe, ${ }^{9-15} \quad 17-24$ except two studies from Asia. ${ }^{16}{ }^{25}$ Eleven studies included patients with MI, ${ }^{9-12} 15-2023$ while the others included patients with ACS with MI and UA. ${ }^{13} 1421222425$ The numbers of patients included in each study varied from 193 to 26641. The mean ages of the included patients ranged from 59 to 67 years, with the percentage of male patients varying from $66 \%$ to $100 \%$. Various symptoms-based anxiety evaluation tools were used to diagnose anxiety. The proportions of patients with anxiety ranged from $9.1 \%$ to $58.2 \%$. Follow-up durations varied from 1 to 10 years after onset of acute coronary events. Potential factors, including demographic characteristics, comorbidities, risk factors for CAD, coronary lesion characteristics and other psychological factors such as depression, that may have confounded the association between anxiety and prognosis after ACS, were adjusted to various extents. The quality of the included studies was generally good, as evidenced by the Newcastle-Ottawa Scale, varying from 7 to 9 stars (online supplementary file 2).

\section{Association between anxiety and mortality in patients with ACS}

The association between anxiety and mortality outcomes for patients with ACS was evaluated in 10 prospective cohorts. ${ }^{10} 12$ 14-17 20212324 Moderate heterogeneity was detected ( $\mathrm{p}$ for Cochrane's $\mathrm{Q}$ test $=0.22, \mathrm{I}^{2}=25 \%$ ). Metaanalysis with a random-effect model showed that anxiety was independently associated with increased mortality risk after acute coronary events (adjusted RR 1.21, 95\% CI 1.07 to $1.37, \mathrm{p}=0.002$; figure $2 \mathrm{~A}$ ). Sensitivity analysis, conducted by excluding one study at a time, retrieved similar results (RR 1.17-1.25, p all <0.05). Subgroup analyses showed that the association between anxiety at baseline and mortality risk in ACS was not significant in studies after adjusting for depression (adjusted RR 0.88, $95 \%$ CI 0.66 to $1.17, \mathrm{p}=0.37, \mathrm{I}^{2}=0 \%$ ), but remained significant in studies without adjusting for depression (adjusted RR $1.28,95 \%$ CI 1.17 to $1.40, \mathrm{p}<0.001, \mathrm{I}^{2}=0 \%$; figure $2 \mathrm{~B}$ ). These findings indicate that depression may confound the association between anxiety and mortality risk after acute coronary events ( $p$ for subgroup difference $=0.01$ ). Moreover, the significant association between anxiety and increased mortality risk was observed in studies including MI patients only (adjusted RR 1.24, 95\% CI 1.09 to 1.41, $\mathrm{p}<0.001, \mathrm{I}^{2}=29 \%$ ), but not in studies with all subtypes of ACS (adjusted RR 0.96, 95\% CI 0.65 to $1.40, \mathrm{p}=0.82$, $\mathrm{I}^{2}=0 \%$; figure 2C). However, the results between the subgroups were not significantly different ( $\mathrm{p}$ for subgroup difference $=0.20$ ).

Association between anxiety and MACEs in patients with ACS

Pooled results with eight prospective cohort

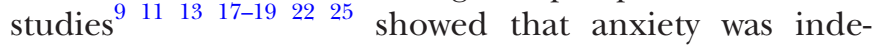
pendently associated with increased risk of MACEs after acute coronary events (adjusted RR 1.47, 95\% CI 1.24 to $1.74, \mathrm{p}<0.001$; figure $3 \mathrm{~A}$ ) with moderate heterogeneity ( $p$ for Cochrane's $Q$ test $=0.16, I^{2}=33 \%$ ). Sensitivity analyses conducted by excluding one study at a time retrieved similar results (RR 1.40-1.54, p all <0.05). Subgroup analyses showed that, although the association between anxiety and increased risk of MACEs remained significant in studies after adjusting for depression (adjusted RR 1.25, $95 \%$ CI 1.04 to $1.52, \mathrm{p}=0.02, \mathrm{I}^{2}=0 \%$ ), the strength of the association was attenuated compared with studies without adjusting for depression (adjusted RR 1.66, 95\% CI 1.31 to $2.10, p<0.001, I^{2}=29 \%$; $p$ for subgroup difference $=0.07$, figure 3B). Moreover, the significant association between anxiety and increased risk of MACEs after acute coronary events was significant in studies with patients with MI (adjusted RR $1.46,95 \%$ CI 1.20 to $1.78 \mathrm{p}<0.001, \mathrm{I}^{2}=17 \%$ ), 


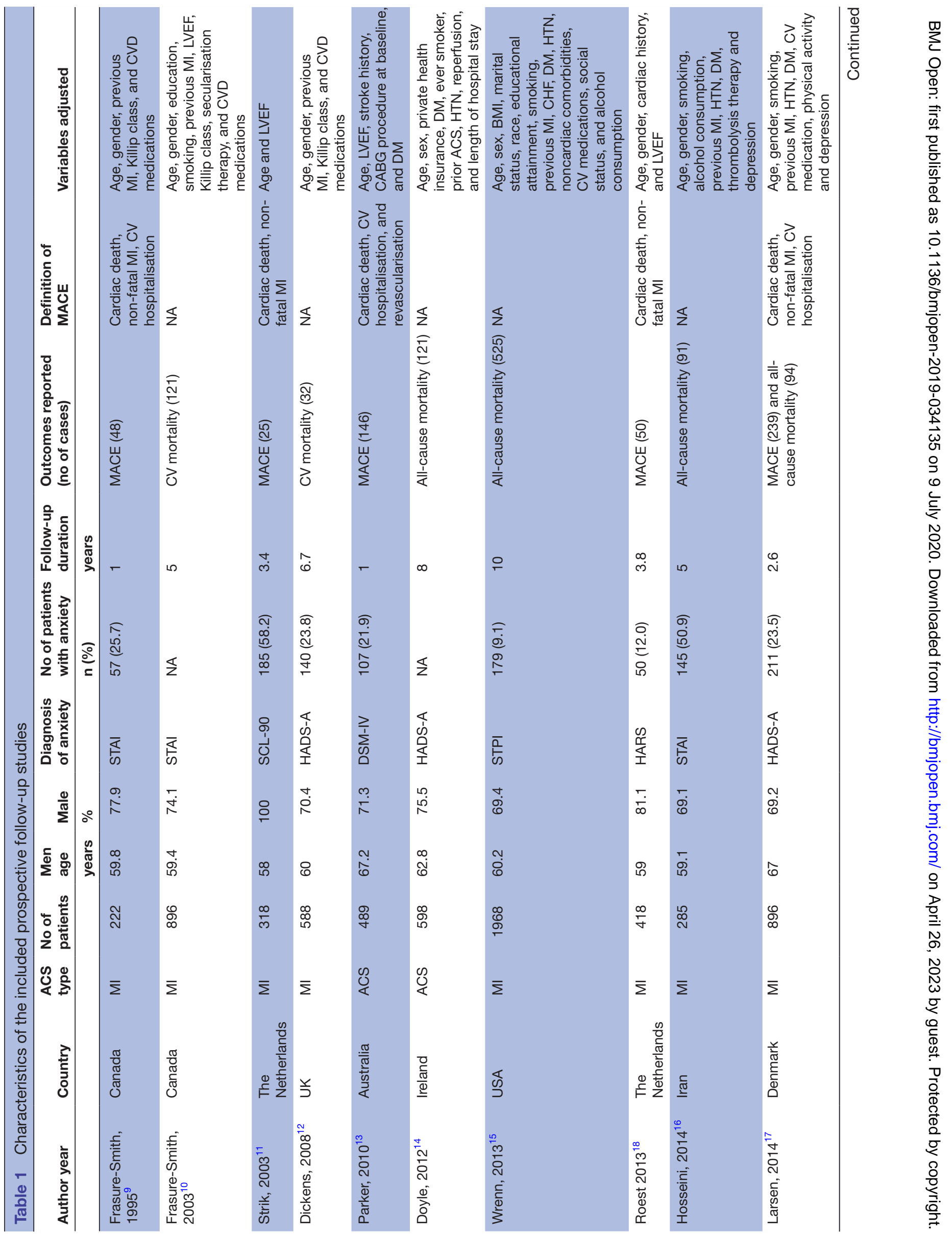




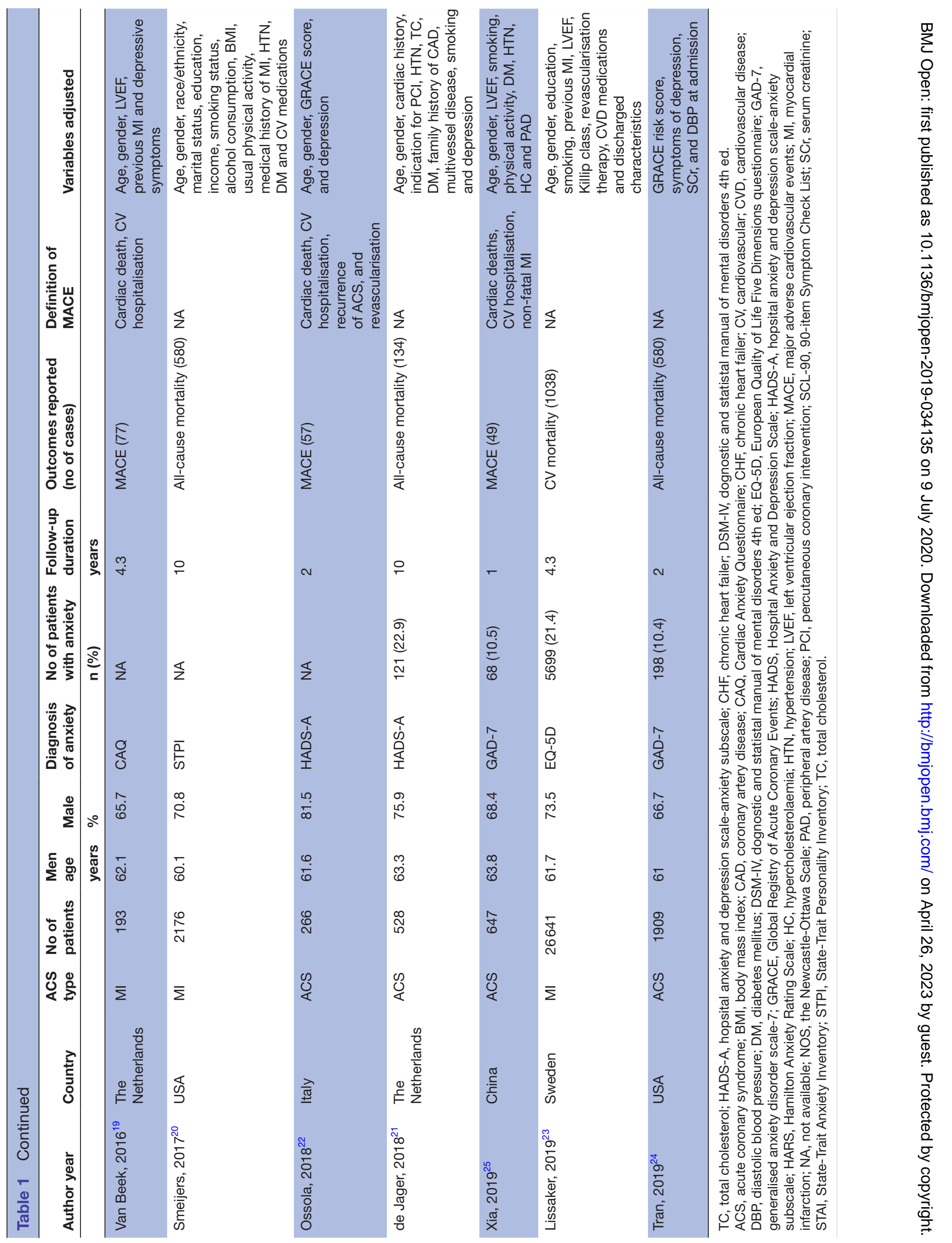




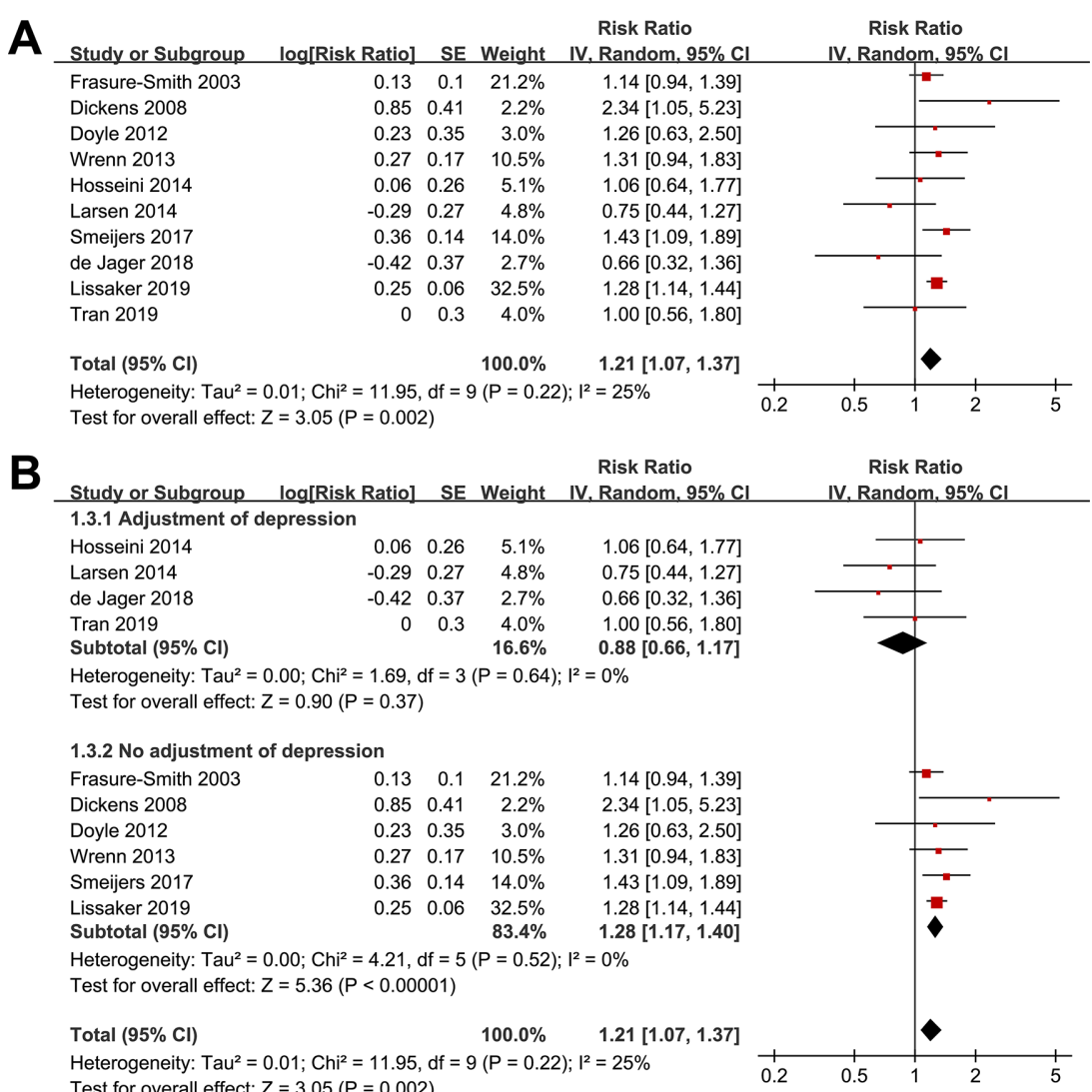

Test for subaroun differences: $\mathrm{Chi}^{2}=6.05 . \mathrm{df}=1(\mathrm{P}=0.01) . \mathrm{I}^{2}=83.5 \%$

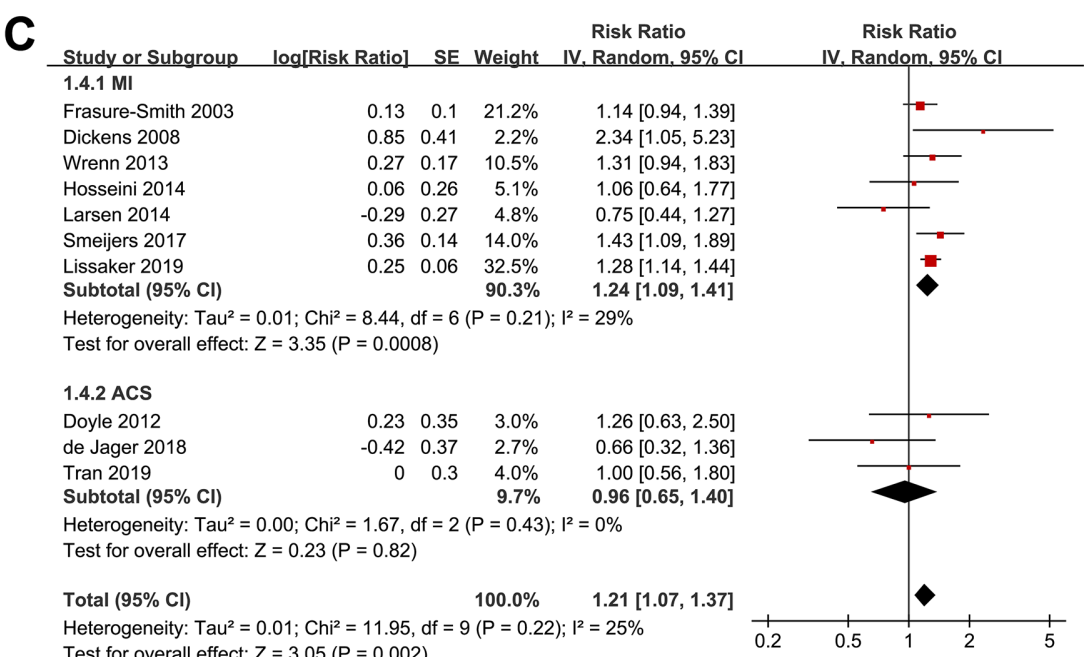

Test for overall effect: $Z=3.05(P=0.002)$

Test for subaroun differences: $\mathrm{Chi}^{2}=1.63 . \mathrm{df}=1(\mathrm{P}=0.20) . \mathrm{I}^{2}=38.8 \%$

Figure 2 Forest plots of the meta-analysis of mortality risk in patients with anxiety after acute coronary events. (A) Forest plots of the main meta-analysis of the association between anxiety and mortality risk in patients with ACS; (B) subgroup analyses after adjusting for depression and (C) subgroup analyses according to the subtypes of ACS. ACS, acute coronary syndrome.

and in studies with all subtypes of patients with ACS (adjusted RR $1.49,95 \%$ CI 1.01 to $2.18, \mathrm{p}=0.04, \mathrm{I}^{2}=65 \%$; figure $3 \mathrm{C}$ ). The results between the subgroups were not significantly different ( $p$ for subgroup difference $=0.94$ ).

\section{Publication bias}

Funnel plots of the meta-analysis of the association between anxiety and risks of mortality and MACEs in patients with ACS are shown in figure $4 \mathrm{~A}$ and $4 \mathrm{~B}$. The funnel plots are symmetrical on visual inspection. Egger's regression tests also showed low risk of publication bias underlining the validity of our meta-analyses for the two outcomes ( $\mathrm{p}=0.527$ and 0.299 , respectively).

\section{DISCUSSION}

Results of our meta-analysis showed that patients with ACS with anxiety at baseline have a $21 \%$ greater risk of mortality and $47 \%$ higher risk of MACEs compared with those without anxiety. However, subgroup analyses showed that concurrent depression may be a significant modifier of the association between anxiety and adverse outcomes 


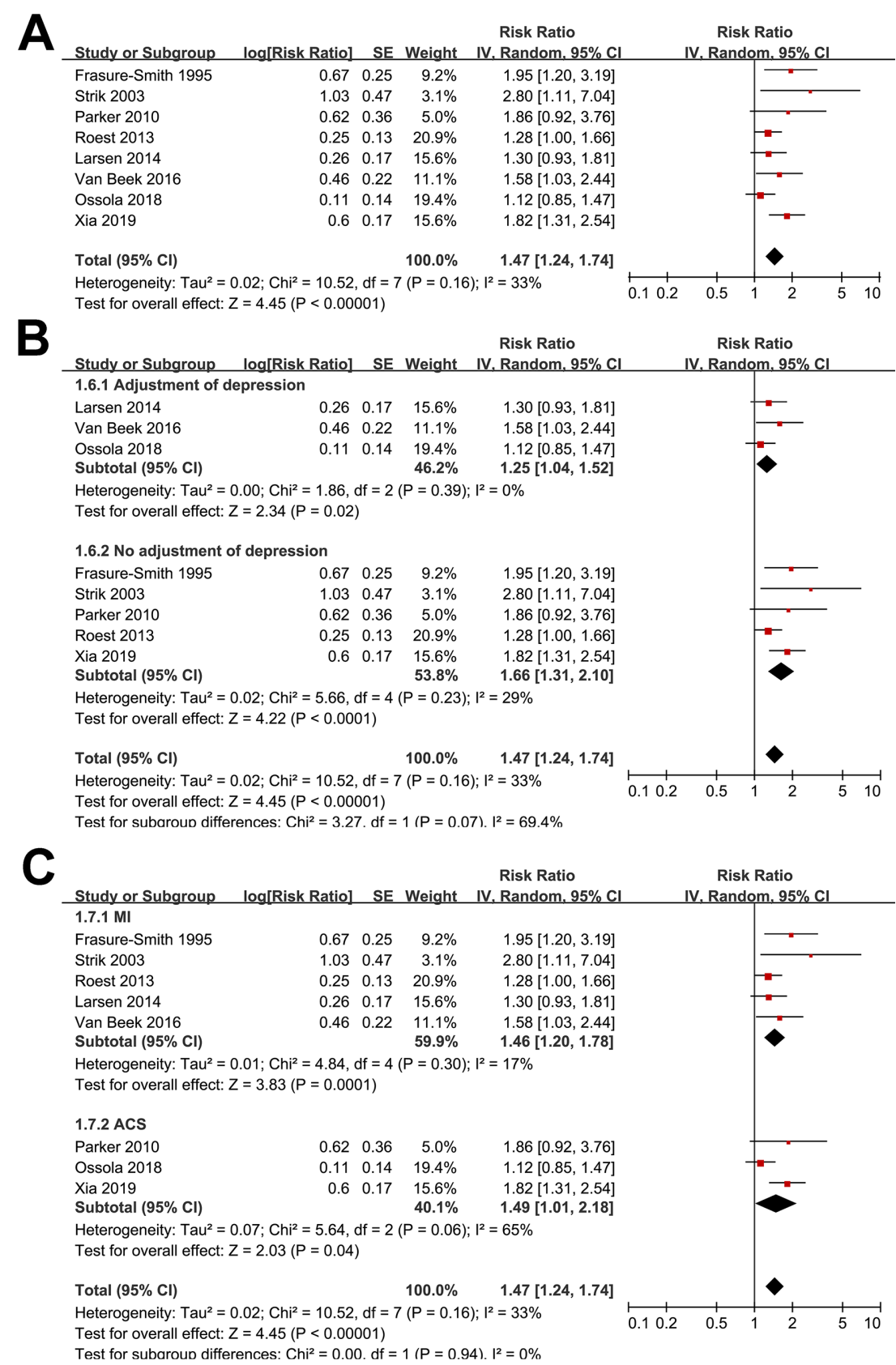

Figure 3 Forest plots of the meta-analysis of MACEs incidence in patients with anxiety after acute coronary events. (A) Forest plots of the main meta-analysis of the association between anxiety and risk of MACEs in patients with ACS; (B) subgroup analyses according to the adjustment of depression and (C) subgroup analyses according to the subtypes of ACS. ACS, acute coronary syndrome; MACEs, mortality and adverse cardiovascular events.

in patients with ACS. Specifically, association between anxiety and increased risk of MACEs in patients with ACS was significantly attenuated in studies that adjusted for depression, and the association between anxiety and mortality risk also became insignificant in these studies. Taken together, these results suggest that patients with ACS with anxiety at baseline have increased risk of mortality and MACEs during follow-up. However, it seems that at least part of the association may be confounded by concurrent depressive symptoms in these patients.

Two previous meta-analyses have been published that evaluated the association between anxiety and prognosis in post-MI patients. Our study has the following important clinical implications compared with the previous ones. First, we expanded the previous study population to include patients with ACS. This is significant because patients with ACS tend to have similar severity of clinical symptoms, complications and exposure to invasive procedures for diagnosis and treatment, which lead to high prevalence of anxiety. Second, we limited our inclusion criteria to prospective follow-up studies with at least 100 patients who were followed for at least 1 year. Moreover, only the adjusted RRs for the associations were included, accounting for potential confounding factors, including demographic factors, CAD risk factors, comorbidities, severity of the coronary lesions and CAD treatment. 


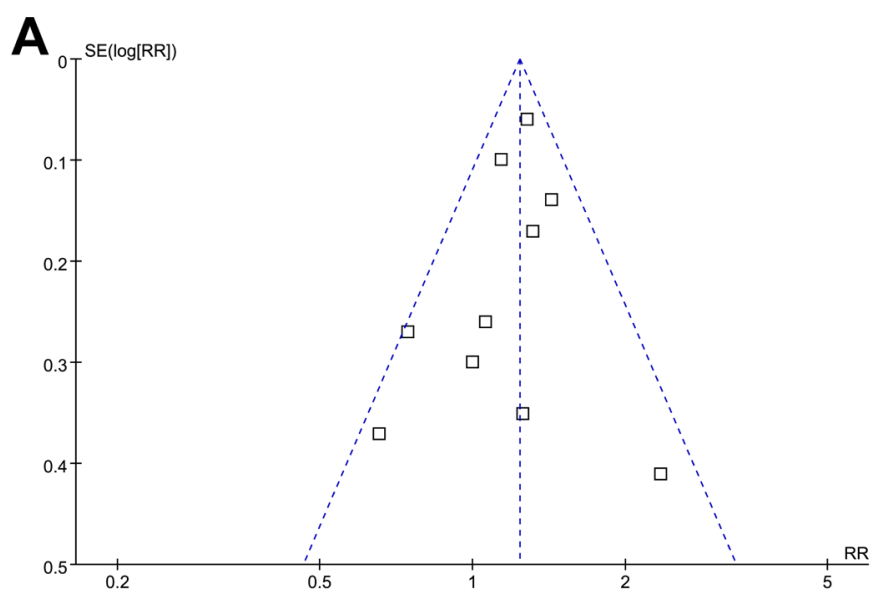

B

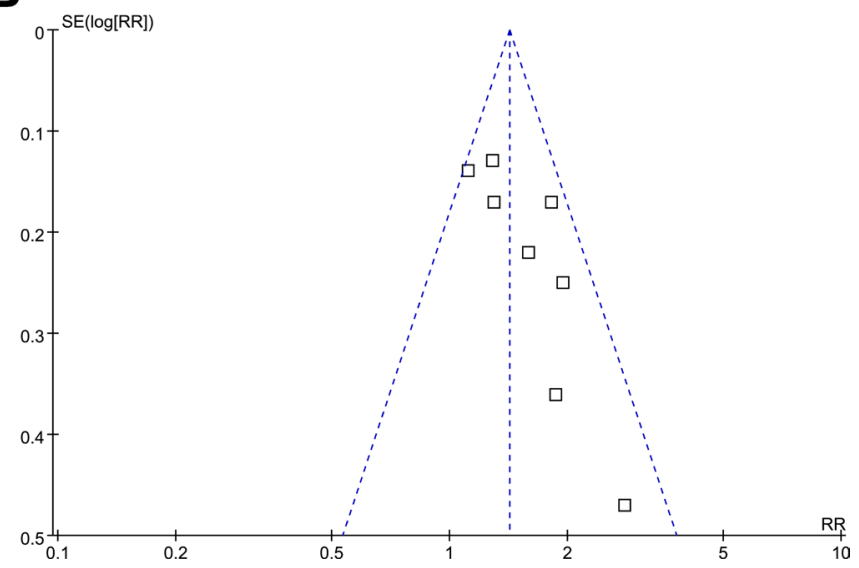

Figure 4 Funnel plots of the meta-analysis of mortality and MACEs in patients with anxiety after acute coronary events; (A) funnel plots of mortality outcome and (B) funnel plots of MACEs outcome. MACEs, mortality and adverse cardiovascular events; RR, risk ratio.

Therefore, our study provides a more reliable result regarding the association between anxiety and prognosis in patients with ACS. Third, by including recently published studies we were able to explore the potential of depression underlying the association between anxiety and poor prognosis in patients with ACS. We found that the potential association between anxiety and increased risk of mortality and MACEs was significantly attenuated or even became insignificant in studies, which had adjusted for depression in their analyses. Previous studies have confirmed that depression is a stronger predictor of poor prognosis in post-MI patients, which may confer a 2-fold to 2.7-fold increased risk of MACEs. ${ }^{36}{ }^{37}$ Moreover, since anxiety and depression are highly correlated psychological disorders ${ }^{28}$ in patients with ACS, depression may be a more important predictor of prognosis than anxiety. During the screening and management of affective disorders in patients with ACS, more efforts should be made to target depression rather than anxiety. However, considering the overlap of clinical manifestations, screening instruments, and pharmacological treatments for anxiety and depression, it would be important to manage emotional disorders in patients with acute coronary events in clinical practice. ${ }^{38-41}$

Some implications could be summarised for clinical practice and future research in this field. The potential pathophysiological basis for the association between anxiety and poor prognosis in patients with ACS may include the following: anxiety is a stressor that might further burden cardiac function. Anxiety might also affect sleep quality, causing disruption of diurnal cycles, thus putting pressure on metabolic pathways leading to further cardiovascular damage. For those diagnosed with anxiety, prescribing anxiolytic drugs may also lead to suppressed respiration and thus further diminish oxygen in the heart. A 'U-shaped' association between anxiety and clinical outcomes has been noticed in some clinical settings. ${ }^{42}$ Patients with mild anxiety may have better compliance, which therefore leads to better clinical prognosis. However, because no data on the severity of anxiety (or dose-response data) was available in the included studies, future studies are needed to investigate whether the association between anxiety and outcomes in patients with ACS varied according to the severity of anxiety. Interestingly, results of subgroup analysis demonstrated that depression may partially confound the association between anxiety and poor prognosis in ACS. The potential mechanisms remain unknown. In view of the common coexistence of anxiety and depression in patients with affective disorders, these findings suggest that management of depression in these patients is important.

Nine different instruments were used as the evaluation tools for anxiety in the included studies. All of the included studies diagnosed anxiety as a binary variable, and the proportions of patients with anxiety at baseline varied considerably. These findings indicate that the diagnostic efficacy and cut-offs for variables anxiety evaluating tools may not be comparable, which may contribute to the heterogeneity. However, since subgroup data (or dose-effect analysis) regarding the severity of anxiety was not reported in either of the included studies, we were unable to determine the potential non-linear association between anxiety and outcomes in patients with ACS. Future studies are needed in this regard.

Despite the clinical implications of our findings, we acknowledge the following limitations of our study. First, the numbers of included studies were limited for the metaanalyses of both MACEs and mortality outcomes, and were even further limited for subgroup analyses. Therefore, results of the subgroup analyses should be interpreted cautiously and validated in the future. Second, although no statistical heterogeneity was detected for our meta-analysis, the instrument and timing of anxiety evaluation may have affected the results, which should be evaluated in the future. Third, we included studies that reported both cardiac mortality and all-cause mortality, and definitions of MACEs varied across the studies, which may have also introduced biases. Fourth, our study is a meta-analysis of observational studies, which do not provide a causative association between anxiety and poor 
outcomes in patients with ACS. Clinical studies evaluating the potential benefits of treatments targeting anxiety in patients with ACS are warranted. Finally, we only included publications in English, and relevant studies published in other languages were omitted.

In conclusion, results of this meta-analysis showed that patients with ACS with anxiety at baseline have an increased risk of mortality and MACEs during a follow-up. At least part of this association may be confounded by concurrent depressive symptoms in these patients. More studies are needed to evaluate the potential interactions between anxiety and depression on clinical outcomes in patients with ACS and to determine the optimal management of these potential affective disorders in patients with acute coronary events.

\section{Author affiliations}

${ }^{1}$ Psychiatric Neuroimaging-Genetic and Comorbidity Laboratory (PNGC_Lab), Tianjin Mental Health Centre, Tianjin Anding Hospital, Tianjin, China

${ }^{2}$ Department of Psychiatric-Neuro-Imaging-Genetics Laboratory, School of Mental of Jining Medical University, Jining, China

${ }^{3}$ Department of Cardiology, Center for Cardiovascular Translational Research, Beijing Key Laboratory of Early Prediction and Intervention of Acute Myocardial Infarction, Peking University People's Hospital, Beijing, China

${ }^{4}$ Health Management Institute, Medical Data Statistical Analysis Center, Medical Big Data Analysis Center, Chinese PLA General Hospital, Beijing, China

${ }^{5}$ Department of Psychiatric-Neuro-Imaging-Genetics Laboratory, Wenzhou Seventh People's Hospital, Wenzhou, China

${ }^{6}$ Department of Psychiatry, Tianjin Fourth Center Hospital, Tianjin, China

Contributors $\mathrm{CZ}$ and XL conceived and designed research. JL, FJ, JS and XG collected and assembled the data. DJ, GC, SC, XL and CZ analysed and interpreted data. JL, FJ, JS and CZ wrote sections of the initial draft. All authors provided substantive suggestions for revision, reviewed and approved final version of the paper.

Funding This work was supported by grants from the National Natural Science Foundation of China (81 871052 to CZ), the Talent Project of Tianjin Anding Hospital(30 000 yuan RMB to CZ), The Key Projects of the Natural Science Foundation of Tianjin, China (17JCZDJC35700 to CZ) and the Tianjin Health Bureau Foundation (2014KR02 to CZ).

Competing interests None declared.

Patient and public involvement Patients and/or the public were not involved in the design, or conduct, or reporting, or dissemination plans of this research.

Patient consent for publication Not required.

Provenance and peer review Not commissioned; externally peer reviewed.

Data availability statement Data are available on reasonable request. The datasets generated and analysed during the present study are available from the corresponding author on reasonable request.

Open access This is an open access article distributed in accordance with the Creative Commons Attribution Non Commercial (CC BY-NC 4.0) license, which permits others to distribute, remix, adapt, build upon this work non-commercially, and license their derivative works on different terms, provided the original work is properly cited, appropriate credit is given, any changes made indicated, and the use is non-commercial. See: http://creativecommons.org/licenses/by-nc/4.0/.

ORCID iD

Chuanjun Zhuo http://orcid.org/0000-0002-3793-550X

\section{REFERENCES}

1 Benjamin EJ, Muntner P, Alonso A, et al. Heart disease and stroke Statistics-2019 update: a report from the American heart association. Circulation 2019;139:e56-28.
2 Amsterdam EA, Wenger NK, Brindis RG, et al. AHA/ACC guideline for the management of patients with non-ST-elevation acute coronary syndromes: Executive summary: a report of the American College of Cardiology/American heart association Task force on practice guidelines. Circulation 2014;2014:2354-94.

3 Levine GN, Bates ER, Blankenship JC, et al. ACC/AHA/SCAl focused update on primary percutaneous coronary intervention for patients with ST-elevation myocardial infarction: an update of the 2011 ACCF/ AHA/SCAI guideline for percutaneous coronary intervention and the 2013 ACCF/AHA guideline for the management of ST-elevation myocardial infarction: a report of the American College of Cardiology/ American heart association Task force on clinical practice guidelines and the Society for cardiovascular angiography and interventions. Circulation 2015;2016:1135-47.

4 Libby P. Mechanisms of acute coronary syndromes and their implications for therapy. N Engl J Med 2013;368:2004-13.

5 Godoy LC, Lawler PR, Farkouh ME, et al. Urgent revascularization strategies in patients with diabetes mellitus and acute coronary syndrome. Can J Cardiol 2019;35:993-1001.

6 Franzon J, Haren MT, Kourbelis C, et al. Recommendations and practices for the screening of depression and anxiety in acute coronary syndrome: a scoping review protocol. JBI Database System Rev Implement Rep 2018;16:1503-10.

7 Lichtman JH, Froelicher ES, Blumenthal JA, et al. Depression as a risk factor for poor prognosis among patients with acute coronary syndrome: systematic review and recommendations: a scientific statement from the American heart association. Circulation 2014:129:1350-69.

8 Abed MA, Kloub MI, Moser DK. Anxiety and adverse health outcomes among cardiac patients: a biobehavioral model. $J$ Cardiovasc Nurs 2014;29:354-63.

9 Frasure-Smith N, Lespérance F, Talajic M. The impact of negative emotions on prognosis following myocardial infarction: is it more than depression? Health Psychol 1995;14:388-98.

10 Frasure-Smith N, Lespérance F. Depression and other psychological risks following myocardial infarction. Arch Gen Psychiatry 2003;60:627-36.

11 Strik JJMH, Denollet J, Lousberg R, et al. Comparing symptoms of depression and anxiety as predictors of cardiac events and increased health care consumption after myocardial infarction. J Am Coll Cardiol 2003;42:1801-7.

12 Dickens C, McGowan L, Percival C, et al. New onset depression following myocardial infarction predicts cardiac mortality. Psychosom Med 2008;70:450-5.

13 Parker GB, Owen CA, Brotchie HL, et al. The impact of differing anxiety disorders on outcome following an acute coronary syndrome: time to start worrying? Depress Anxiety 2010;27:302-9.

14 Doyle F, Conroy R, McGee H. Differential predictive value of depressive versus anxiety symptoms in the prediction of 8year mortality after acute coronary syndrome. Psychosom Med 2012;74:1-6.

15 Wrenn KC, Mostofsky E, Tofler GH, et al. Anxiety, anger, and mortality risk among survivors of myocardial infarction. Am J Med 2013:126:1107-13.

16 Hosseini SH, Ghaemian A, Mehdizadeh E, et al. Levels of anxiety and depression as predictors of mortality following myocardial infarction: a 5-year follow-up. Cardiol J 2014;21:370-7.

17 Larsen KK, Christensen B, Nielsen TJ, et al. Post-Myocardial infarction anxiety or depressive symptoms and risk of new cardiovascular events or death: a population-based longitudinal study. Psychosom Med 2014;76:739-46.

18 Roest AM, Heideveld A, Martens EJ, et al. Symptom dimensions of anxiety following myocardial infarction: associations with depressive symptoms and prognosis. Health Psychol 2014;33:1468-76.

19 Van Beek MHCT, Zuidersma M, Lappenschaar M, et al. Prognostic association of cardiac anxiety with new cardiac events and mortality following myocardial infarction. Br J Psychiatry 2016;209:400-6.

20 Smeijers L, Mostofsky E, Tofler GH, et al. Anxiety and anger immediately prior to myocardial infarction and long-term mortality: characteristics of high-risk patients. J Psychosom Res 2017;93:19-27.

21 de Jager TAJ, Dulfer K, Radhoe S, et al. Predictive value of depression and anxiety for long-term mortality: differences in outcome between acute coronary syndrome and stable angina pectoris. Int J Cardiol 2018;250:43-8.

22 Ossola P, Gerra ML, De Panfilis C, et al. Anxiety, depression, and cardiac outcomes after a first diagnosis of acute coronary syndrome. Health Psychol 2018;37:1115-22.

23 Lissåker CT, Norlund F, Wallert $\mathrm{J}$, et al. Persistent emotional distress after a first-time myocardial infarction and its association to late 
cardiovascular and non-cardiovascular mortality. Eur J Prev Cardiol 2019;26:1510-8.

24 Tran H, Byatt N, Erskine N, et al. Impact of anxiety on the postdischarge outcomes of patients discharged from the hospital after an acute coronary syndrome. Int J Cardiol 2019;278:28-33.

25 Xia K, Wang L-F, Yang X-C, et al. Comparing the effects of depression, anxiety, and comorbidity on quality-of-life, adverse outcomes, and medical expenditure in Chinese patients with acute coronary syndrome. Chin Med J 2019;132:1045-52.

26 Roest AM, Martens EJ, Denollet J, et al. Prognostic association of anxiety post myocardial infarction with mortality and new cardiac events: a meta-analysis. Psychosom Med 2010;72:563-9.

27 Celano CM, Millstein RA, Bedoya CA, et al. Association between anxiety and mortality in patients with coronary artery disease: a meta-analysis. Am Heart J 2015;170:1105-15.

28 de Jonge P, Ormel J, van den Brink RHS, et al. Symptom dimensions of depression following myocardial infarction and their relationship with somatic health status and cardiovascular prognosis. Am J Psychiatry 2006;163:138-44.

29 Stroup DF, Berlin JA, Morton SC, et al. Meta-Analysis of observational studies in epidemiology: a proposal for reporting. meta-analysis of observational studies in epidemiology (moose) group. JAMA 2000;283:2008-12.

30 Higgins J, Green S. Cochrane Handbook for systematic reviews of interventions. Version 5.1.0. The Cochrane Collaboration, 2011. www.cochranehandbook.org

31 Wells GA, Shea B, O'Connell D, et al. The Newcastle-Ottawa scale (NOS) for assessing the quality of nonrandomised studies in metaanalyses, 2010. Available: http://www.ohri.ca/programs/clinical_ epidemiology/oxford.asp
32 Huang Y, Cai X, Mai W, et al. Association between prediabetes and risk of cardiovascular disease and all cause mortality: systematic review and meta-analysis. BMJ 2016;355:i5953.

33 Higgins JPT, Thompson SG. Quantifying heterogeneity in a metaanalysis. Stat Med 2002;21:1539-58.

34 Patsopoulos NA, Evangelou E, loannidis JPA. Sensitivity of betweenstudy heterogeneity in meta-analysis: proposed metrics and empirical evaluation. Int J Epidemiol 2008;37:1148-57.

35 Egger M, Davey Smith G, Schneider M, et al. Bias in meta-analysis detected by a simple, graphical test. BMJ 1997;315:629-34.

36 Meijer A, Conradi HJ, Bos EH, et al. Prognostic association of depression following myocardial infarction with mortality and cardiovascular events: a meta-analysis of 25 years of research. Gen Hosp Psychiatry 2011;33:203-16.

37 van Melle JP, de Jonge P, Spijkerman TA, et al. Prognostic association of depression following myocardial infarction with mortality and cardiovascular events: a meta-analysis. Psychosom Med 2004;66:814-22.

38 Celano CM, Villegas AC, Albanese AM, et al. Depression and anxiety in heart failure: a review. Harv Rev Psychiatry 2018;26:175-84.

39 Cohen BE, Edmondson D, Kronish IM. State of the art review: depression, stress, anxiety, and cardiovascular disease. Am J Hypertens 2015;28:1295-302.

40 Silverman AL, Herzog AA, Silverman DI. Hearts and minds: stress, anxiety, and depression: unsung risk factors for cardiovascular disease. Cardiol Rev 2019;27:202-7.

41 Małyszczak K, Rymaszewska J. Depression and anxiety in cardiovascular disease. Kardiol Pol 2016;74:603-9.

42 Mykletun A, Bjerkeset O, Overland S, et al. Levels of anxiety and depression as predictors of mortality: the HUNT study. Br J Psychiatry 2009;195:118-25. 